\title{
Survey on Condition and Orthodontic Treatment of Maxillary Lateral Incisors in Patients with Cleft Lip and Palate
}

\author{
Mayuko Uchino $^{1)}$, Hidehito Arima ${ }^{2)}$, Hiroshi Kobayashi ${ }^{3)}$, Junya Nagata ${ }^{4)}$, \\ Toshihiko Yasumura ${ }^{1)}$, Takenobu Ishii ${ }^{1)}$, Haruyo Miyazaki ${ }^{1)}$, \\ Teruo Sakamoto ${ }^{2)}$, Kunihiko Nojima ${ }^{2)}$ and Kenji Sueishi ${ }^{1,2)}$ \\ ${ }^{1)}$ Department of Orthodontics, Tokyo Dental College, \\ 2-9-18 Kanda-Misakicho, Chiyoda-ku, Tokyo 101-0061, Japan \\ 2) Department of Orthodontics, Tokyo Dental College, \\ 1-2-2 Masago, Mihama-ku, Chiba 261-8502, Japan \\ ${ }^{3)}$ Nikkori Orthodontic Clinic \\ 3F, 1-2-50 Wakamiya, Nirasaki-shi, Yamanashi 407-0015, Japan \\ 4) NAGATA ORTHODONTIC OFFICE \\ 6F Meijiyakyoto Bldg. 78-80 Higashiirunakajima-cho, Sanjokawara-machi, Nakagyo- \\ ku, Kyoto 604-8004, Japan
}

Received 12 February, 2019/Accepted for Publication 30 August, 2019 Published Online in J-STAGE 10 June, 2020

\begin{abstract}
The aim of this study was to investigate the condition of the maxillary lateral incisors and evaluate the methods used for cleft closure in patients with cleft lip and palate, including the treatment of the maxillary lateral incisors. A total of 214 patients (260 clefts) with alveolar clefts who had started Phase II treatment and entered the maintenance period at the Department of Orthodontics at Tokyo Dental College, Chiba Hospital, between 1975 and 2014 were included. Panoramic, intraoral, and occlusal radiographs, as well as intraoral photographs and medical records, were used to investigate cleft classification, the presence or absence and location of maxillary lateral incisors, and frequency and treatment method for peg lateral incisors in the cleft region. There were more unilateral cleft cases $(78.5 \%)$ than bilateral cleft cases. The prevalence of congenital absence of the maxillary lateral incisors was similar between unilateral $(53.0 \%)$ and bilateral cases $(53.3 \%)$. Peg laterals occurred frequently, with $89.9 \%$ occurring in unilateral cases. The maxillary lateral incisors were more commonly found in the secondary than in the primary palate. The number of non-extraction cases was larger than that of extraction cases, regardless of cleft type or the location of the peg laterals. In many cases, the peg laterals were treated with non-extraction and space closure or crown modification. These results suggest that, depending on their condition, the peg laterals should be preserved as much as possible in devising a treatment plan.
\end{abstract}

Key words: Cleft lip and palate - Orthodontic treatment -

Congenital absence - Maxillary lateral incisors - Peg laterals 


\section{Introduction}

Anomalies in the number and size of the teeth adjacent to the alveolar cleft region occur frequently in patients with cleft lip and palate $^{1,4,16,18,19)}$. Congenital absence of the maxillary lateral incisors in the cleft region is often involved in such cases. Even if the maxillary lateral incisors are present, they may be pegshaped and therefore require extraction, the alternative usually involving crown modification or leaving undisturbed to achieve tooth alignment. In cases where extraction is to be performed, however, orthodontic movement of the adjacent teeth or a prosthetic procedure is necessary to close the resulting space. Several options have been proposed for treating cases of alveolar cleft. Understanding the anatomical anomaly and the effect of maxillary lateral incisor absence in the cleft region in the dentition is important in managing occlusion and treatment outcomes. The purpose of this study was to investigate the condition of the maxillary lateral incisors and evaluate the methods used for cleft closure in patients with cleft lip and palate, including the treatment of the maxillary lateral incisors.

\section{Materials and Methods}

The protocol of this study was reviewed and approved by the Tokyo Dental College Ethics Review Committee (Approval number, 699). A total of 214 patients (260 clefts) who started receiving orthodontic treatment and entered the maintenance management phase over a period of 40 years between 1975 and 2014 at Tokyo Dental College, Chiba Hospital were included in the study.

The panoramic, intraoral, and occlusal radiographs, intraoral photographs, and medical records of each patient were obtained. Cleft classification, presence or absence and location of maxillary lateral incisors, and frequency and treatment method for peg laterals in the cleft region were determined.

\section{Cleft classification}

The ratio of unilateral and bilateral clefts was determined.

\section{Frequency of missing maxillary lateral incisors and peg laterals}

The ratio of presence to absence of maxillary lateral incisors in the alveolar cleft region was determined in unilateral and bilateral cleft patients. The frequency of peg laterals was determined in patients with maxillary lateral incisors. In unilateral cleft patients, the presence/absence of lateral incisors was categorized into the following 4 groups: absentpresent, absent-absent, present-absent, and present-present for the cleft and non-cleft sides, respectively. In bilateral cleft patients, the presence/absence of lateral incisors was categorized into the following 3 groups: both sides absent (both-absent), one side present (one side-present), and both sides present (both-present). The percentage of cases that were categorized into each group was calculated.

Any tooth in the cleft region was considered a lateral incisor, regardless of its morphology. If a tooth was missing from the cleft region, then the lateral incisor was considered congenitally missing. When there were supernumerary teeth in the cleft region, the largest tooth was treated as the lateral incisor. Supernumerary teeth were excluded from this survey.

The percentage of the lateral incisor crown width to the central incisor crown width was calculated. Lateral incisors with a percentage $<69.9 \%$ were classified as peg laterals ${ }^{11)}$. When it was difficult to distinguish between deciduous teeth and permanent peg laterals, even after considering the crown and root morphology of the tooth, medical records, and progress images, the decision was made based on age. If the patient was 5 to 12 years old, with a missing permanent tooth germ in the alveolar cleft region, the erupted tooth was treated as a deciduous lateral incisor. For patients aged 12 years or older, the erupted tooth was treated as the permanent peg lateral incisor ${ }^{17)}$. 




Fig. 1 Presence or absence and morphology of maxillary lateral incisors in alveolar cleft region in unilateral cleft patients

\section{Location of maxillary lateral incisors}

Whether the maxillary lateral incisors were located in the primary or secondary palate was determined.

\section{Extraction or non-extraction and treatment method for peg laterals}

The ratio of extraction to non-extraction methods used in treating peg laterals in the alveolar cleft was determined in both unilateral and bilateral cleft patients; it was also determined according to the involvement of peg laterals in the primary or secondary palate.

The treatment methods for peg laterals in unilateral and bilateral clefts were categorized into the following 6 groups: extraction + space closure, extraction + prosthetic replacement, non-extraction + space closure, non-extraction + crown modification, non-extraction + space retention, or other.

\section{Statistical analysis}

The chi-squared $\left(\chi^{2}\right)$ test was used for the statistical analysis of proportional data. The level of statistical significance was set at $\mathrm{p}<0.05$.

\section{Results}

\section{Cleft classification}

The total of 214 cases reviewed comprised 168 of unilateral cleft $(78.5 \%)$ and 46 of bilateral cleft $(21.5 \%)$.
Table 1 Percentage of patients with unilateral cleft who had missing maxillary lateral incisors

\begin{tabular}{lcc}
\hline \hline $\begin{array}{c}\text { cleft side-non } \\
\text { cleft side }\end{array}$ & Number & $\%$ \\
\hline present-present & 75 & 44.6 \\
absent-present & 70 & 41.7 \\
absent-absent & 19 & 11.3 \\
present-absent & 4 & 2.4 \\
\hline Total & 168 & 100 \\
\hline
\end{tabular}

\section{Frequency of missing maxillary lateral incisors and peg laterals}

Among the 168 cases of unilateral cleft, the maxillary lateral incisors were absent in 89 $(53.0 \%)$. Of the total number of maxillary lateral incisors, 71 (89.9\%) were peg laterals (Fig. 1). Table 1 shows the classification of unilateral cleft patients based on the presence or absence of lateral incisors on the cleft and non-cleft sides.

Among the 46 cases of bilateral cleft comprising 92 alveolar clefts, the maxillary lateral incisors were absent in $49(53.3 \%)$. Of the total number of maxillary lateral incisors, 29 $(67.4 \%)$ were peg laterals (Fig. 2). Table 2 shows the classification of bilateral cleft patients based on the presence or absence of lateral incisors at the alveolar cleft.

The $\chi^{2}$ test was used to determine if there were differences in the presence or absence of teeth in the alveolar cleft between unilat- 


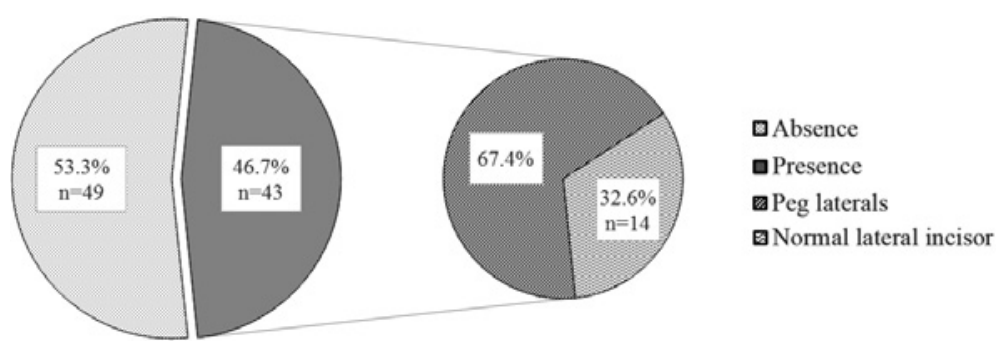

Fig. 2 Presence or absence and morphology of maxillary lateral incisors in alveolar cleft region in bilateral cleft patients

Table 2 Percentage of patients with bilateral clefts who had missing maxillary lateral incisors

\begin{tabular}{lcc}
\hline \hline & Number & $\%$ \\
\hline both-absent & 19 & 41.3 \\
both-present & 16 & 34.8 \\
one side-present & 11 & 23.9 \\
\hline Total & 46 & 100 \\
\hline
\end{tabular}

eral and bilateral cleft cases. No significant difference in the occurrence of missing maxillary lateral incisors was identified between unilateral and bilateral cleft cases $\left(\chi^{2}=0.00192, \mathrm{df}=1, \mathrm{p}>0.05\right)$. The $\chi^{2}$ test was also performed to reveal differences in the peg anatomy of maxillary lateral incisors in the cleft between unilateral and bilateral cleft patients. A significantly higher occurrence of peg laterals was observed in unilateral than in bilateral clefts $\left(\chi^{2}=9.48\right.$, df $\left.=1, \mathrm{p}<0.05\right)$.

\section{Location of maxillary lateral incisors}

Among the 79 maxillary lateral incisors found in unilateral clefts, $67(84.8 \%)$ were found in the secondary palate. Among the 43 maxillary lateral incisors found in bilateral clefts, $36(83.7 \%)$ were found in the secondary palate (Table 3 ).

No significant difference was observed in the location of the maxillary lateral incisors between unilateral and bilateral clefts according to the $\chi^{2}$ test $\left(\chi^{2}=0.025, \mathrm{df}=1, \mathrm{p}>0.05\right)$. Lateral incisors were observed more com- monly in the secondary than in the primary palate.

\section{Extraction or non-extraction and treatment method for peg laterals}

Among the 71 peg laterals found in unilateral clefts, $49(69.0 \%)$ were not extracted. Among the 29 peg laterals found in bilateral clefts, $16(55.2 \%)$ were not extracted (Table 4). No significant difference was identified in the use of non-extraction between unilateral and bilateral clefts $\left(\chi^{2}=1.73, \mathrm{df}=1, \mathrm{p}>0.05\right)$.

For the peg laterals found in unilateral clefts, the most common treatment method was non-extraction + space closure (23 teeth, $32.4 \%)$. For peg laterals found in bilateral clefts, the most common treatment method was non-extraction + crown modification (9 teeth, $31.0 \%$; Table 4).

Three peg laterals found in the primary palate were treated with extraction $(27.3 \%)$ and 8 with non-extraction $(72.7 \%)$. Thirtytwo peg laterals found in the secondary palate were treated with extraction $(36.0 \%)$ and 57 with non-extraction $(64.0 \%)$.

\section{Discussion}

This study showed that the maxillary lateral incisors in the alveolar cleft region were absent in approximately 53\% of both unilateral and bilateral cleft cases. In past reports, the absence of maxillary lateral incisors was predicted to be more common in bilateral 
Table 3 Position of maxillary lateral incisors

\begin{tabular}{lccc}
\hline \hline & $\begin{array}{c}\text { Primary palate } \\
\text { number of lateral incisors (\%) }\end{array}$ & $\begin{array}{c}\text { Secondary palate } \\
\text { number of laterals incisors (\%) }\end{array}$ & Total (\%) \\
\hline Unilateral cleft patients & $12(15.2)$ & $67(84.8)$ & $79(100)$ \\
Bilateral cleft patients & $7(16.3)$ & $36(83.7)$ & $43(100)$ \\
\hline
\end{tabular}

Table 4 Extraction or non-extraction and treatment method for peg laterals

\begin{tabular}{|c|c|c|c|c|c|}
\hline & & $\begin{array}{l}\text { Unilatera } \\
\text { number of }\end{array}$ & $\begin{array}{l}\text { ft patients } \\
\text { laterals }(\%)\end{array}$ & $\begin{array}{r}\text { Bilateral } \\
\text { number of }\end{array}$ & $\begin{array}{l}\text { patients } \\
\text { laterals }(\%)\end{array}$ \\
\hline \multirow{2}{*}{ Extraction } & prosthetic replacement & $15(21.1)$ & \multirow{2}{*}{$22(31.0)$} & $6(20.7)$ & \multirow{2}{*}{$13(44.8)$} \\
\hline & space closure & $7(9.9)$ & & $7(24.1)$ & \\
\hline \multirow{4}{*}{ Non extraction } & crown modification & $15(21.1)$ & \multirow{4}{*}{$49(69.0)$} & $9(31.0)$ & \multirow{4}{*}{$16(55.2)$} \\
\hline & space closure & $23(32.4)$ & & $6(20.7)$ & \\
\hline & space retention & $8(11.3)$ & & $1(3.4)$ & \\
\hline & other & $3(4.2)$ & & $0(0)$ & \\
\hline & Total & $71(100)$ & & $29(100)$ & \\
\hline
\end{tabular}

clefts, which are more severe than unilateral clefts $^{4,12)}$. This finding was not supported by the present results, however, which showed no difference in frequency between the two types. Although the relationship between tooth absence and cleft patterns has been investigated in various studies ${ }^{4,16,18,19)}$, the results have been inconsistent. This is most likely due to lateral incisors in the cleft region often being associated with agenesis, dislocation, extraction during surgery, impaction, or even oversight of a history of tooth extraction. This indicates the need for information to be shared among cooperating hospitals so that each patient's medical history may be more accurately determined.

In the present study, congenital absence of the maxillary lateral incisors consistent with alveolar cleft was observed frequently in both unilateral (absent-present, 41.7\%) and bilateral (both-absent, $41.3 \%$ ) cleft patients. Tooth absence in the cleft region has shown correlations with surgical history, or a deficiency in blood supply or mesenchymal cells ${ }^{4,12}$. In the present study, the maxillary lateral incisors were also absent on the non-cleft side in $13.7 \%$ of unilateral cases. Therefore, it is possible that other factors contributed to the congenital absence of the maxillary lateral incisors. Earlier studies ${ }^{14,19,21,23)}$ have reported that mutations in the PAX9 and MSX1 genes cause tooth agenesis and cleft lip and palate, suggesting that some cases of alveolar clefts and tooth agenesis may have genetic etiologies. In this study, 4 unilateral cleft patients were noted in whom the lateral incisors were present on the cleft side but missing on the non-cleft side (present-absent, 2.4\%). In these patients, genetic effects were probably more pronounced than were the effects of the alveolar cleft.

The frequency of peg laterals was higher in unilateral clefts, at $89.9 \%$, than in bilateral clefts, at $67.4 \%$. Similar to the relationship between the frequency of congenital absence of the maxillary lateral incisors and the severity of alveolar clefts, the ratio of peg laterals in the cleft region varies ${ }^{2,7,18)}$. It is unclear if the 
frequency of peg laterals is higher in unilateral or in bilateral clefts. Reports have shown that the occurrence of peg laterals may be related to the compromised growth potential seen in patients with cleft lip and palate, or to external factors such as surgical intervention or nutrition ${ }^{8,13)}$.

Regarding the position of the maxillary lateral incisors, similar to the findings of past reports ${ }^{10,24)}$, many teeth were observed in the secondary palate $(84.8 \%$ in unilateral and $83.7 \%$ in bilateral clefts). These results suggest that the maxillary lateral incisor primordia may be located in the medial nasal or maxillary process in patients with cleft lip and palate $^{6,12)}$.

The present findings regarding the treatment used for peg laterals showed that the non-extraction method was the most common in cases of both unilateral and bilateral cleft. Non-extraction was selected when the inclination of the maxillary central incisors and the sagittal relationship of the dental arches were within acceptable proportions, and the discrepancy between tooth size and arch length was small. Nevertheless, extraction was performed in a larger number of bilateral than in unilateral clefts. This may have been because bilateral clefts cause greater complications than do unilateral clefts. In patients with cleft lip and palate, the standard of self-cleansing in the oral cavity tends to be poor due to abnormalities in tooth development, malocclusion, or surgical procedure-induced scarring, any of which can restrict adequate movement of the lips and palate. One report showed that the prevalence of caries was higher among patients with cleft lip and palate than among the general population ${ }^{3)}$. The presence of caries can increase the need for tooth extraction if the prognosis is poor. Extraction of a tooth close to a cleft should be avoided whenever possible, however, as extraction causes alveolar bone resorption at the site and gingival recession of the tooth adjacent to the defect. Hence, it is important to initiate education on oral hygiene as early as possible as a preventive measure in such cases.
In terms of the treatment used for the peg laterals, the present results revealed that nonextraction + space closure was the most common type in cases of unilateral cleft, while non-extraction + crown modification was the most common in cases of bilateral cleft. To determine whether peg laterals should remain untreated or undergo crown modification, esthetic factors and the anterior ratio need to be considered on an individual basis.

Where extraction had been undertaken, space closure was rare in cases of unilateral cleft because of difficulties associated with achieving symmetry of tooth numbers, the occlusal relationship, or matching of the dental midline with the facial midline. It is important for both the patient and the orthodontist to understand that the period of orthodontic treatment will be longer where treatment is to involve extraction + space closure. In this study, prosthetic replacement accounted for $20 \%$ of both unilateral and bilateral cleft cases. Taking into account the position of the canines in the cleft ${ }^{5}$, prosthetic replacement was selected for cases where a Class III relationship could be improved by camouflage treatment ${ }^{15}$; when the symmetry of dentition could be maintained, even with a unilateral defect of the maxillary lateral incisors; or when there was a desire to maintain tongue space $^{9,15)}$. On the other hand, some studies have reported the advantages of using alveolar bone grafting prior to eruption of the maxillary canines ${ }^{8}$, or as early as possible, to achieve permanent dentition without prosthodontics $^{20)}$ and to preserve the maxillary lateral incisors ${ }^{22)}$.

According to the results of the present survey, some patients were not followed up for prosthetic replacement. In Japan, prosthetic replacement following peg lateral extraction is usually performed after the patient reaches adulthood (18 years of age). Therefore, when Phase II of a treatment plan is completed while the patient is still young, it will be necessary for follow up with regular check-ups to be continued until the final prosthesis is placed so that long-term stability of the dentition and occlusion may be maintained. In this survey, 
no cases involving dental implants after extraction of peg laterals were identified. This was most likely because, in Japan, dental implants constitute a marked financial burden for patients, and in many cases, re-bone grafting is required prior to implantation ${ }^{8)}$.

In terms of the use of treatment methods that depended on the position of the maxillary lateral incisors, non-extraction was common for peg laterals found in both the primary and secondary palates in the present study. Therefore, the rate of non-extraction treatment was high for peg laterals in the alveolar cleft, regardless of cleft type or location. While formulating a treatment plan, it is important to comprehensively evaluate the occlusal condition, in addition to the condition of the maxillary lateral incisors in the alveolar cleft.

The limitations of this study include a small sample size and inadequate management in terms of the final prosthesis. Hence, longterm maintenance after orthodontic treatment is crucial. Furthermore, the relationship between the maxillary lateral incisors and supernumerary teeth in the alveolar cleft, the extraction or non-extraction of other teeth, and anomalous tooth numbers in areas other than the alveolar cleft should be investigated in the future.

\section{Conclusion}

Congenital absence of the maxillary lateral incisors in the alveolar cleft region was frequently observed. The maxillary lateral incisors were often present in the secondary palate, and the frequency of microdontia was high. Non-extraction of peg laterals was the most common type of treatment. The treatment plan was usually determined taking into consideration the condition of both the occlusal and maxillary lateral incisors. Further investigation is needed to identify trends in treatment method in more detail.

\section{References}

1) Baek SH, Kim NY (2007) Congenital missing permanent teeth in Korean unilateral cleft lip and alveolus and unilateral cleft lip and palate patients. Angle Orthod 77:88-93.

2) Celikoglu M, Buyuk SK, Sekerci AE, Cantekin K, Candirli C (2015) Maxillary dental anomalies in patients with cleft lip and palate: a cone beam computed tomography study. J Clin Pediatr Dent 39:183-186.

3) Chopra A, Lakhanpal M, Rao NC, Gupta N, Vashisth S (2014) Oral health in 4-6 years children with cleft lip/palate: a case control study. N Am J Med Sci 6:266-269.

4) Deepti A, Muthu MS, Kumar NS (2007) Root development of permanent lateral incisor in cleft lip and palate children: a radiographic study. Indian J Dent Res 18:82-86.

5) Freitas JA, Garib DG, Oliveira M, Lauris Rde C, Almeida AL, Neves LT, Trindade-Suedam IK, Yaedú RY, Soares S, Pinto JH (2012) Rehabilitative treatment of cleft lip and palate: experience of the Hospital for Rehabilitation of Craniofacial Anomalies-USP (HRAC-USP) part 2: Pediatric dentistry and orthodontics. J Appl Oral Sci 20:268-281.

6) Garib DG, Rosar JP, Sathler R, Ozawa TO (2015) Dual embryonic origin of maxillary lateral incisors: clinical implications in patients with cleft lip and palate. Dent Press J Orthod 20:118-125.

7) Germec CD, Nur YRB, Bulut FN, Aksoy A (2018) Dental anomalies in different types of cleft lip and palate: Is there any relation? J Craniofac Surg 29:1316-1321.

8) Gillgrass TJ, MacDonald JP, Mossey PA, Welbury RR (2014) The impact of alveolar bone grafting on cleft lip and palate: a literature review. South Eur J Orthod Dentofacial Res 1:15-22.

9) Hang WM, Village W (2007) Treatment of missing lateral incisors. Am J Orthod Dentofacial Orthop 125:18A-19A.

10) Kelley MD, Sheldon P, Daniela GG (2012) Is missing maxillary lateral incisor in complete cleft lip and palate a product of genetics or local environment? Angle Orthod 82: 959-963.

11) Kondo S, Matsuno M, Futagami C, Hanamura H, Kanazawa E (2010) Analysis of heredity factors in the morphological variation of the maxillary lateral incisor by twin model. Jinruigaku Zasshi 118:1-10.

12) Lourenço RL, Teixeira DNL, Costa B, Ribeiro GM (2003) Dental anomalies of the permanent lateral incisors and prevalence of hypodontia outside the cleft area in complete 
unilateral cleft lip and palate. Cleft Palate J 40: 172-175.

13) McCance AM, Roberts-Harry D, Sherriff M, Mars M, Houston WJB (1990) A study model analysis of adult unoperated Sri Lankans with unilateral cleft lip and palate. Cleft Palate J 27: 146-154.

14) Mossey PA, Little J, Munger RG, Dixon MJ, Shaw WC (2009) Cleft lip and palate. Lancet 374:1773-1785.

15) Oosterkamp BC, Dijkstra PU, Remmelink HJ, Oort RP, Sandham A (2010) Orthodontic space closure versus prosthetic replacement of missing upper lateral incisors in patients with bilateral cleft lip and palate. Cleft Palate J 47: 591-596.

16) Paradowska-Stolarz A, Dubowik M, Szeląg J, Kawala B (2014) Dental anomalies in the incisor-canine region in patients with cleft lip and palate - literature review. Dev Period Med 18:66-69.

17) Peterka M, Peterková R, Likovský Z (1996) Timing of exchange of maxillary deciduous and permanent teeth in boys with three types of orofacial clefts. Cleft Palate J 33:318-323.

18) Rullo R, Festa VM, Rullo F, Addabbo F, Chiodini P, Vitale M, Perillo L (2015) Prevalence of dental anomalies in children with cleft lip and unilateral and bilateral cleft lip and palate. Eur J Paediatr Dent 16: 229-232.

19) Sato M, Baba Y, Haruyama N, Higashihori N, Tsuji M, Suzuki S, Moriyama K (2016) Clinicostatistical analysis of congenitally miss- ing permanent teeth in Japanese patients with cleft lip and/or palate. Orthod Waves 75: 41-45.

20) Semb G, Ramstad T (1999) The influence of alveolar bone grafting on the orthodontic and prosthodontic treatment of patients with cleft lip and palate. Dent Update 26:60-64.

21) Stockton DW, Das P, Goldenberg M, D’Souza RN, Patel PI (2000) Mutation of PAX9 is associated with oligodontia. Nat Genet 24:18-19.

22) Tereza GPG, Santos MACD, Winckler VPSV, Almeida ALPF, Dalben GDS (2018) The maxillary lateral incisor in the rehabilitation of cleft lip and palate. J Appl Oral Sci 26: e20170125.

23) Van den Boogaard MJ, Dorland M, Beemer FA, van Amstel HK (2000) MSX1 mutation in associated with orofacial clefting and tooth agenesis in humans. Nat Genet 24:342-343.

24) Yatabe MS, Garib DG, Janson G, Poletto RS, Ozawa TO (2013) Is the presence of Simonart's band in patients with complete unilateral cleft lip and palate associated with the prevalence of missing maxillary lateral incisors? Am J Orthod Dentofacial Orthop 144:649-653.

\section{Correspondence:}

Dr. Mayuko Uchino

Department of Orthodontics, Tokyo Dental College, 2-9-18 Kanda-Misakicho, Chiyoda-ku, Tokyo 101-0061, Japan E-mail: monoda@tdc.ac.jp 